\title{
Artículos
}

\section{Lenguajes de marcas aplicados a la transformación de estructuras documentales}

Resumen: Este artículo se ocupa de las tecnologías CSS (cascading style sheet) y Xslt (extensible style sheets language: transformations). Se trata de un trabajo de carácter práctico, sencillo y demostrativo más que un estudio teórico o exhaustivo. La idea que se pretende transmitir es que la unidad básica de gestión de información vuelve a ser el documento, con su marca de documento digital. La tecnología en la web se orienta hacia el control absoluto de su ciclo vital, funcionalidades y componentes estructurales. Este artículo presenta Xslt como una de las opciones más interesantes para controlar la estructura de los documentos digitales mediante transformaciones.

Palabras clave: CSS (cascading style sheet), Documento digital, DOM (document object model), Hojas de estilo, html (hypertext markup language), Internet Explorer, sgml (standard generalized markup language), XSL (extensible style sheets language), Xslt (extensible style sheets language: transformations), Xslf(extensible style sheets language: formatting), xml (extensible markup language).

Title: Markup languages applied to the transformation of documentary structures.

Abstract: This article addresses CSS (cascading style sheet) and Xslt (extensible style sheets language: transformations) technologies, from a practical, straightforward and demonstrative perspective, rather than attempting to provide a theoretical or exhaustive study. The central idea is that the basic unit of information management again becomes the document, marked up as a digital document. Web technology is directed at an absolute control over its life-cycle, functionalities and structural elements. This article presents Xslt as one of the most interesting options for controlling digital documents' structure through transformations.

Keywords: CSS (cascading style sheet), Digital document, DOM (document object model), Style sheets, html (hypertext markup language), Internet Explorer, sgml (standard generalized markup language), XSL (extensible style sheets language), Xslt (extensible style sheets language: transformations), Xslf (extensible style sheets language: formatting), xml (extensible markup language).

Rosa Piñero, Antonio de la. "Lenguajes de transformación de estructuras aplicados al intercambio de documentación". En: El profesional de la información, 2001, enero-febrero, v. 10, n. 1-2, pp. 4-22.

\section{Introducción}

¿De dónde viene la idea de tratar un documento digital como una estructura arbórea de nodos? En realidad se trata de la evolución lógica de una de las áreas de estudio clásicas de la informática: el desarrollo de aplicaciones para la generación y edición automática de documentos digitales. Este campo abarca un amplio espectro de programas de todo tipo (procesadores de textos sencillos, sistemas de ayuda basados en hipertexto, producción automática de bases de datos, edición, diseño, etc.) dirigidas a todos los tipos de usuarios: principiantes, profesionales o programadores.

Históricamente, la evolución del procesamiento de textos se puede esquematizar en los siguiente pasos: durante la década de los 60 el sistema utilizado era simple: tras generar el documento se aplicaba el formato deseado. Por lo general, la salida de este texto era impresa, y aquel tenía asociado, junto al dato propiamente dicho, la descripción deseada (llamada reproducción) que era convertida por el programa en una presentación. Algunas de las notaciones utilizadas en esta época para la reproducción siguen estando hoy en día en vigencia (con modificaciones, claro está): rtf (rich text format) o troff.

\section{«Para gestionar información de forma eficaz es necesario con- tar con un sistema que permita estructurarla lógicamente»}

Poco tiempo después de que este sistema empezara a tener éxito, apareció el marcado de formato: se procedía a marcar el texto con una serie de etiquetas o códigos. Con la aparición de sistemas wysiwyg (what you see is what you get) se produjo una separación. Por un lado se desarrollaron lenguajes de etiquetas más complejos, por otro, se unió la potencia de los sistemas de reproducción a la capacidad de las nuevas interfaces. El producto final de esta opción son los actuales procesadores de texto (AmiPro, Pagemaker, Word, WordPerfect, etc.).

Por Antonio de la Rosa Piñero, consultor e ingeniero de programación, Wisdom B. V., Assen, Holanda. antonio@wisdom.nl

Original recibido el 9-9-00

Aceptación definitiva: 26-10-00 


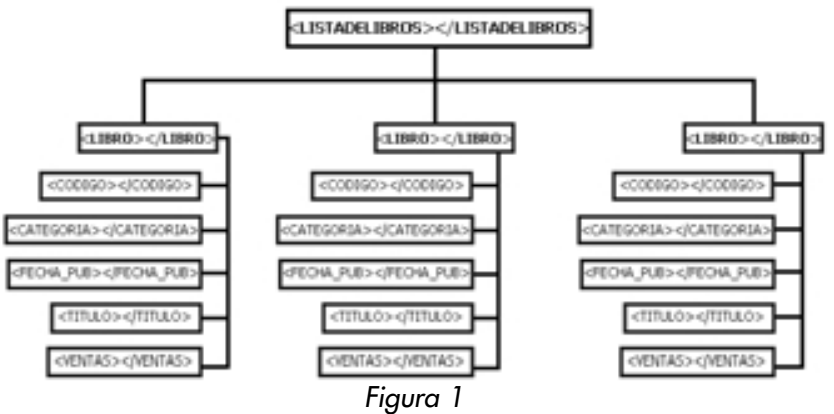

La proliferación de diversos formatos generó un problema: la información tenía diferentes maneras de representarse. Dentro de este contexto apareció, a finales de los 60, sgml. Lo interesante de esta evolución es constatar cómo en un momento determinado se produjo una separación entre presentación y estructuración.

El siguiente paso en la web ha sido asimilar la idea de que para gestionar información de forma eficaz es necesario contar con un sistema que permita estructurarla lógicamente, ya sea de manera tabular o arbórea, puesto que esa ordenación facilitará la automatización de las operaciones que se realicen sobre ella: recuperación, intercambio, integración, etc. Por otra parte, mantener una estructura de datos rígida dentro de un modelo abierto, como es el caso de la www, es un contrasentido.

\section{Bibliografía recomendada}

Cascading style sheets, level 2 Css2 specification. W3C recommendation, 12 de mayo, 1998.

http://www.w3.org/TR/REC-CSS2/

Cascading style sheets home page.

http://www.w3.org/Style/CSS/

Extensible markup language $(\mathrm{xml}) \quad 1.0$ (second edition). W3C recommendation, 6 de octubre, 2000.

http://www.w3.org/TR/2000/REC-xm/20001006

Francis, Brian, et al. IE5 dynamic html programmer's reference. Chicago (Illinois): Wrox Press Inc., 1999. Isbn I86I00I746.

Kay, Michael. Xslt programmer's reference. Chicago (Illinois): Wrox Press Inc., 2000. Isbn I86I003I 29.

Xsl transformations (Xs/t), version I.0. W3C recomendation, 16 de noviembre, 1999. http://www.w3.org/TR/xs/t

El profesional de la información está abierto a todos los bibliotecarios, documentalistas y profesionales de la información, así como a las empresas y organizaciones del sector para que puedan exponer sus noticias, productos, servicios, experiencias y opiniones.

Dirigir todas las colaboraciones para publicar a:

El profesional de la información

Apartado 32.280

08080 Barcelona

Fax: +34-934250029

epi@sarenet.es

De ahí el éxito del formato xml (extensible markup language), válido para la representación digital de documentos de cualquier tipo. Para trabajar con él es necesario estructurar la información. Éste es un proceso muy sencillo dentro del campo de la documentación, ya que la mayoría de "materiales" con los que se trabaja es susceptible de ser dividida en componentes. Así, un libro tiene título, capítulos, índices o un artículo resumen, apartados, subapartados, notas al pie, etc. Al mismo tiempo, cada una de estas partes tiene a su vez párrafos, frases, imágenes, etc. Xml denomina a todos ellos integrantes.

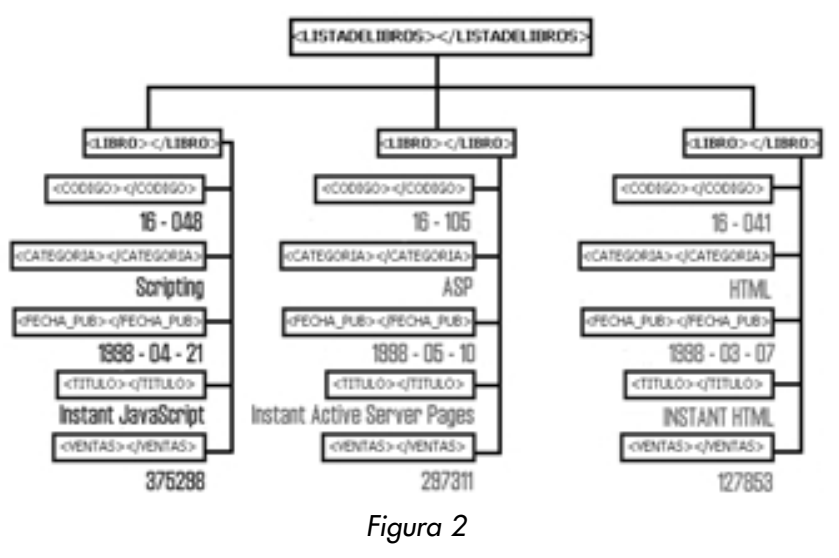

La siguiente tabla muestra una lista de libros formateada en xml:

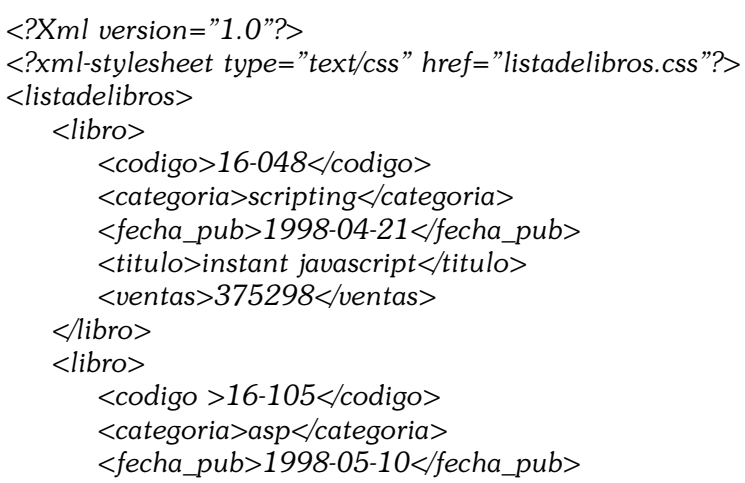




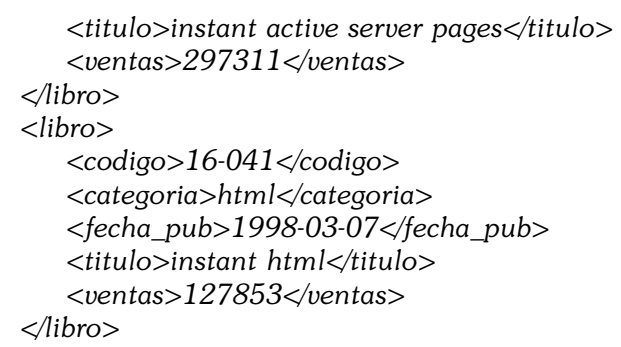

Esto lo podríamos traducir gráficamente al árbol de etiquetas presentado en la figura 1. El cual, algo más desarrollado, genera el árbol de contenidos de la figura 2.

Éste es el estado de la cuestión actual por lo que se refiere a documentación digital en la www y xml.

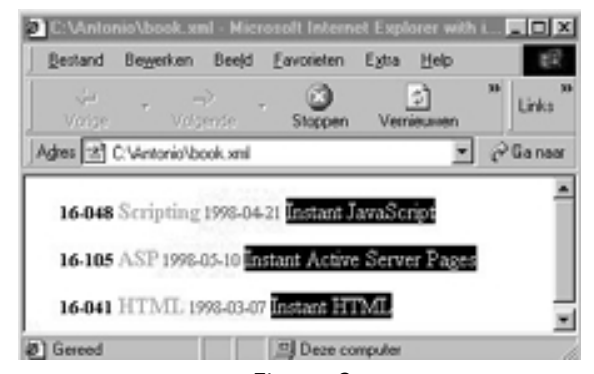

Figura 3

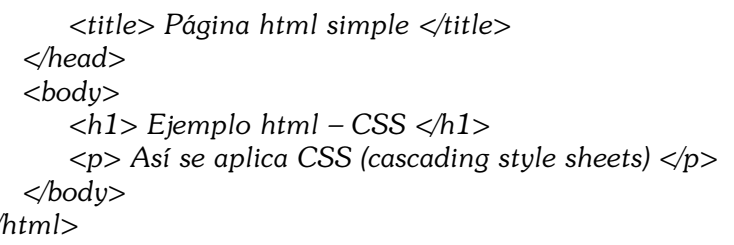

Para hacer que el contenido del elemento $\langle h l\rangle$ aparezca en azul, se podría usar la siguiente regla CSS:

h1 \{color:blue\}

Una regla CSS consta de dos componentes principales: un selector ('hl') y una declaración ('color:blue') la cual, a su vez, tiene dos partes: propiedad ('color') y valor ('blue'). El código anterior podría verse como una hoja de estilo muy simple; combinada con otras (su característica fundamental ${ }^{1}$ es que pueden combinarse) servirá para determinar la presentación final del documento.

La especificación html 4.0 define cómo se deben aplicar las reglas CSS a los documentos html: tanto dentro del mismo documento como mediante una hoja de estilo externa. Para incluirla internamente se usa el elemento <style $>$ :

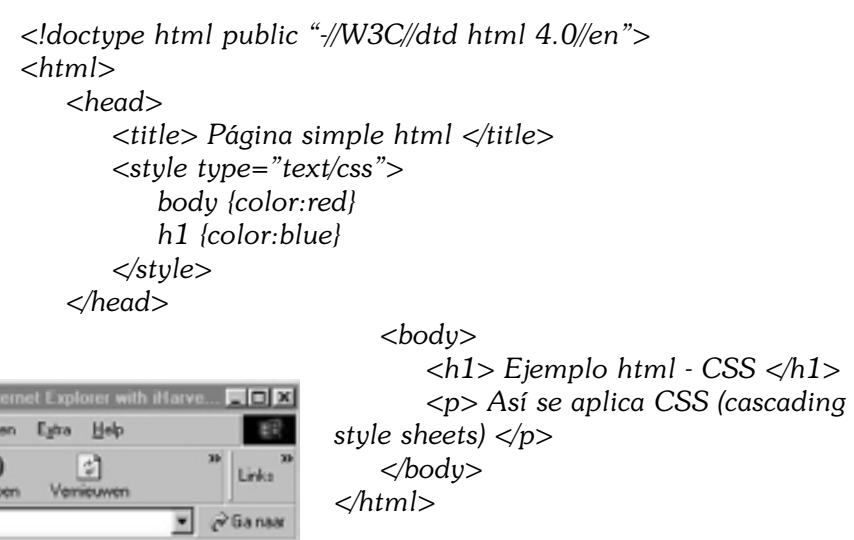
sheets) y de xslt (extensible style sheets language: transformations), las dos especificaciones en las que se centra este estudio. Como se intentará demostrar, las implicaciones de su planteamiento de trabajo van mucho más allá de la simple presentación de datos.

\section{css}

El ejemplo bajo estas líneas es un documento html a partir del cual se van a introducir algunos conceptos sobre esta clase de hojas de estilo:

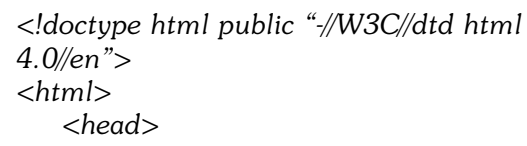

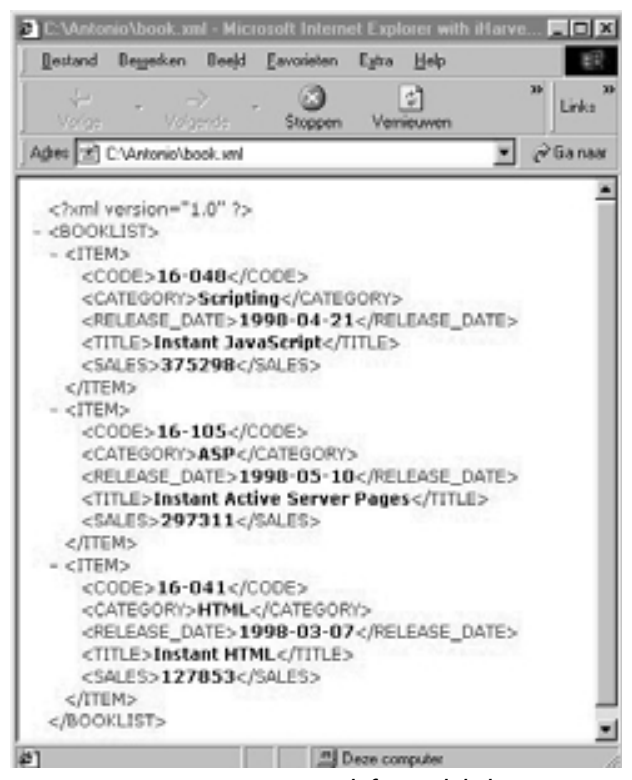

Figura 4. Apariencia por defecto del documento book.xml en IE5
La hoja de estilo (en este caso se corresponde con el elemento $<$ style $>$ ) contiene dos reglas: la primera hace que el elemento $<$ body $>$ adopte por defecto el color rojo mientras que la segunda hace posible que el contenido de $<h l>$ aparezca en azul. No se especifica ningún color para $<p>\mathrm{y}$ por lo tanto heredará el color de su elemento padre (aquel en el cual está contenido): <body $>$. $<h l>$ es también hijo de $<$ body $>$ pero en este caso se aplica la segunda regla de la hoja de estilo. 'Color' es sólo una de las aproximadamente 100 propiedades incluidas en CSS-2. En el siguiente código se utilizan algunas otras: 
$<$ !doctype $h$ tml public "-//W3C//dtd html 4.0//en">

$<h t m l>$

$<$ head $>$

$<$ title $>$ Página simple $\mathrm{html}</$ title $>$

$<$ style type $=$ "text/css">

body \{

font-family:"Gill Sans" sans-serif;

font-size:12pt

margin:3em ; \}

h1 \{color:blue\}

$</$ style $>$

$<$ head $>$

$<$ body $>$

$<$ h1 $>$ Ejemplo html - CSS $</ \mathrm{h} 1>$

$<p>$ Así se aplica CSS (cascading style sheets) $</ p>$ $</$ body $>$

$<$ html $>$

El primer punto a comentar es que hay varias declaraciones agrupadas en un bloque de código delimitado por llaves $(\{\ldots\})$, separadas con (;), aunque la última puede aparecer también seguida por (:). La primera declaración asociada con el elemento $<$ body $>$ fija el tipo de letra en gill sans. Si no está disponible, el navegador usará sansserif, que es uno de los genéricos que todos los navegadores "conocen". Los hijos del elemento $<$ body $>$ heredarán el valor de la

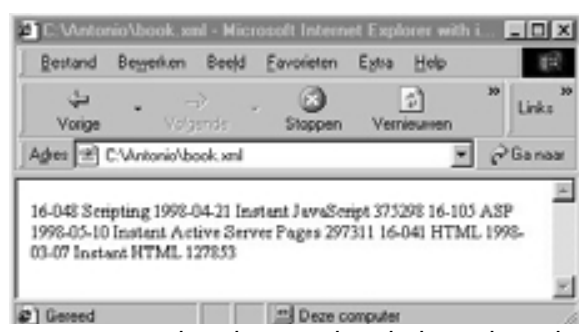

Figura 5. Book.xml asociado a la hoja de estilo Xslt expresada en el código anterior propiedad font-family si no especifican los suyos propios. La segunda declaración fija el tamaño de la letra del elemento $<$ body $>$ en 12 puntos $^{2}$. La tercera usa una unidad de dimensión relativa, la unidad "em", y se refiere a la medida de la letra; en este caso, los márgenes alrededor de dicho elemento serán tres veces el tamaño de la letra (por lo tanto, 36 puntos).

1. Elementos-objeto (selectores). El siguiente ejemplo es un poco más complicado. El código define un estilo para todos los elementos $\langle p\rangle: 14$ puntos, color verde, letra arial; y otro para todos los que presenten un atributo "class" con el valor " miclase": 12 puntos, color azul, letra courier:

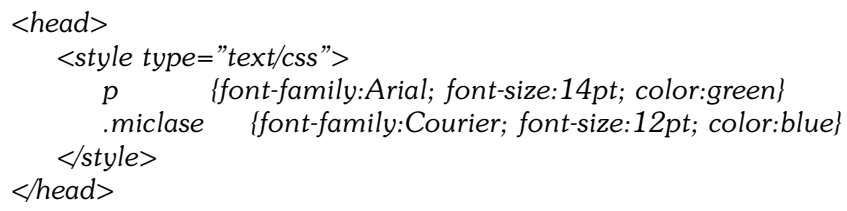

Como se ha visto antes, CSS permite definir un selector o elemento-objeto del estilo a aplicar, usando el nombre de dicho elemento, como se acaba de hacer con $\langle p\rangle$. Es posible ser más precisos a la hora de especificar los selectores indicando su forma de anidamiento. En este otro ejemplo el estilo se aplica solamente a los elementos $\langle e m>$ anidados dentro de $\langle p\rangle$ :
El siguiente código es ligeramente diferente, puesto que separa los nombres de los selectores con una coma. Con esta sintaxis, el estilo se aplicará a todos los elementos $\langle p>$ y a todos los $\langle e m\rangle$ :

p, em \{font-family:Arial; font-size:14pt; color:green\}

2. Clases-objeto. El ejemplo anterior también definía un estilo para una clase llamada miclase. Para indicar que es una clase propietaria ${ }^{3}$, se antecede el nombre con un punto:

miclase \{font-family:Courier; font-size:12pt; color:blue\}

Ahora se puede asignar este estilo a cualquier elemento usando el atributo (class) de la siguiente forma:

$<p$ class $=$ "miclase" $>$ Texto $</ p>$

a. Estilos dinámicos. Usar clases objeto, como en el ejemplo anterior, es una técnica muy popular cuando se utiliza dhtml (dynamic html). La razón es que un script puede cambiar el estilo de un elemento en respuesta a un determinado evento. Si se definen varias clases, sólo habrá que ajustar la propiedad className del elemento en cuestión a la clase apropiada usando el script. En el siguiente ejemplo se cambia la propiedad className de un elemento $\langle p>$ en respuesta a un even-

to onmouseover:

<style type="text/css">

grande\{font-family:Arial; font-size:24pt; font-weight:bold\} .pequeifont-family:Courier; font-size:12pt; font-

weight:normal \}

$</$ style $>$

$<p$ class $=$ "peque"

id ="miEncabez"onmouseover="hazGrande();"onmouseout $=$ "haz Peque();" $>$ Texto $</ p>$

$<$ script language="JScript" >

function hazGrande()

\{

document.all('miEncabez').className = 'grande';

function hazPeque() l

document.all('miEncabez').className = 'peque'; $</$ script $>$

b. Hojas de estilo enlazadas. El ejemplo anterior utiliza una sección <style $>$ dentro de un documento html para definir la información relativa a su presentación. Sin embargo, también se puede vincular una hoja de estilo independiente con un documento html usando tanto el elemento <link> como la instrucción de estilo@import.Los siguientes ejemplos enlazan al documento html una hoja independiente llamada miestilo.css:

$<$ link rel="stylesheet" type="text/css" href="miestilo.css"> 
El elemento $<$ link $>$ especifica: heet”.

— El tipo de archivo con el que se enlaza: "styles"href".

— El tipo de hoja de estilo: "text/css".

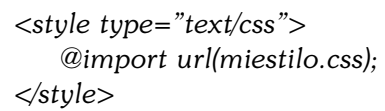

Es siempre recomendable usar hojas de estilo externas por dos motivos fundamentales: pueden modificarse sin tocar el documento fuente html y es posible compartirlas entre diferentes documentos.

3. Usar CSS con documentos xml. A las aplicaciones documentales les resulta más interesante el uso que se pueda hacer de CSS a partir de documentos xml. ¿Cómo se trabaja con esto? No es posible usar bloques de código delimitados por el elemento <style $>$ ya que no tiene sentido en xml. Sucede lo mismo con $<$ link $>$; el navegador presentará el contenido de ambos como simple texto; al fin y al cabo éste es el planteamiento tras xml: cada elemento significa lo que su creador decida.

\section{«Salvando las distancias se po- dría decir que, en un futuro pró- ximo, un documento digital en la www se considerará como una pequeña base de datos»}

a. La instrucción de proceso $\mathrm{xml}$-stylesheet. La solución al problema es usar una instrucción de proceso $x \mathrm{ml}$. Este lenguaje define el elemento <? xml...? $>$ como una instrucción de proceso que se usa para dar órdenes al parser o procesador xml. Una versión especial de esa instrucción de proceso se usa para enlazar documentos xml con las hojas de estilo que contienen la información sobre su presentación:

$<$ ?xml-stylesheet type="text/css" href="miestilo.css"?>

El parser xml buscará la hoja de estilo en la dirección especificada en el atributo href y la usará para dar formato al contenido del documento xml. También se pueden definir diferentes hojas de estilo usando varias instrucciones $<$ ? xml-stylesheet.. $>$. En ese caso los estilos se fusionan y, en caso de duplicación, los últimos en aplicarse tienen precedencia.

b. Un ejemplo. El siguiente código muestra cómo una hoja de estilo CSS puede servir para controlar la presentación de un documento xml, llamado book. $x m l^{4}$, que es una lista de tres libros:

$<? x m l$ version $=$ "1.0"? $>$

$<$ ?xml-stylesheet type="text/css" href="listadelibros.css"?>

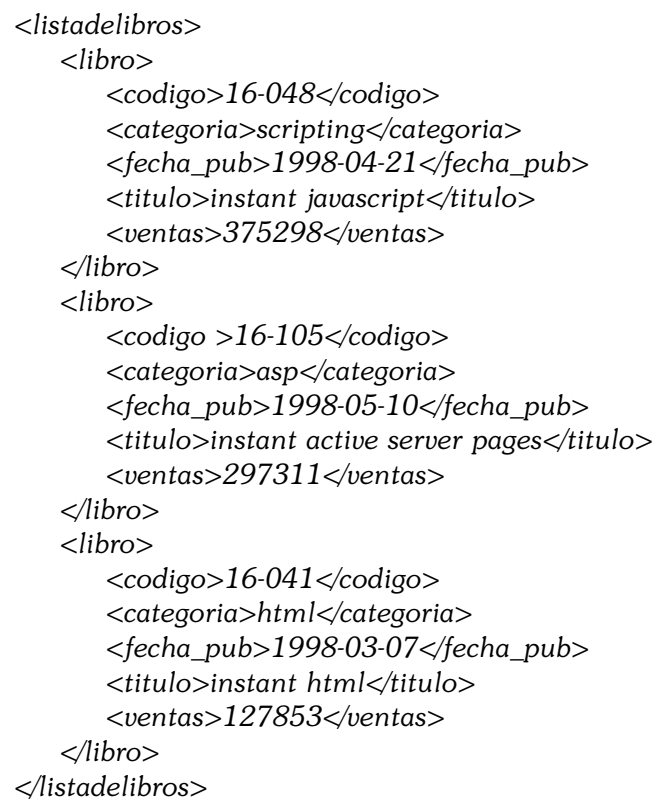

Se puede observar que la segunda línea es la instrucción de proceso que especifica la hoja de estilo llamada listadelibros.css. A continuación se muestra su contenido:

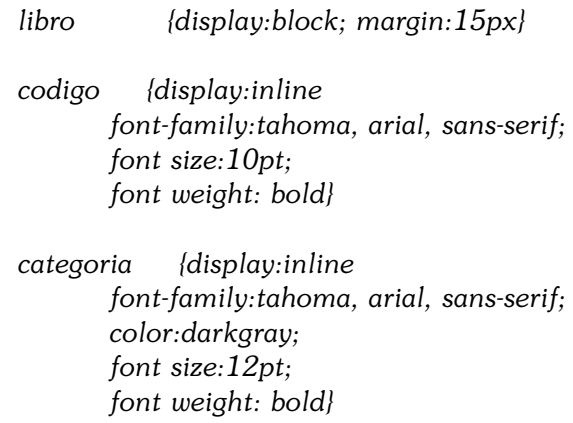

fecha_pub \{display:inline

font-family:tahoma, arial, sans-serif;

color:red;

font size: 10pt\}

titulo \{display:inline

font-family:tahoma, arial, sans-serif;

font size: $12 \mathrm{pt}$;

color:white

background-color:black\}

ventas \{display:none

Para cada uno de los elementos del documento fuente xml (excepto <listadelibros $>$ ), se especifica un conjunto de propiedades de estilo. Para el elemento $<$ libro $>$ se define como display:block. Por lo tanto, cada <libro > aparecerá formando un bloque ${ }^{5}$ separado de los demás por un salto de línea. Esto es análogo a lo que se consigue con el elemento html $<$ div $>$. También

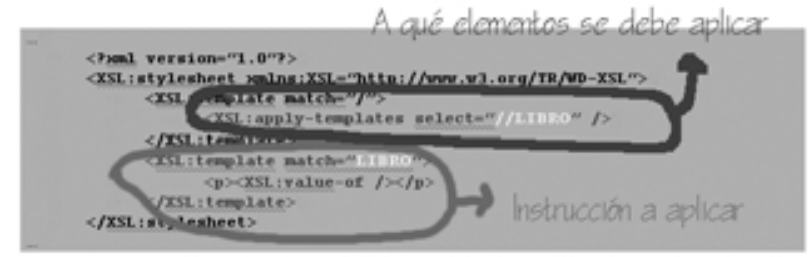

Figura 6 


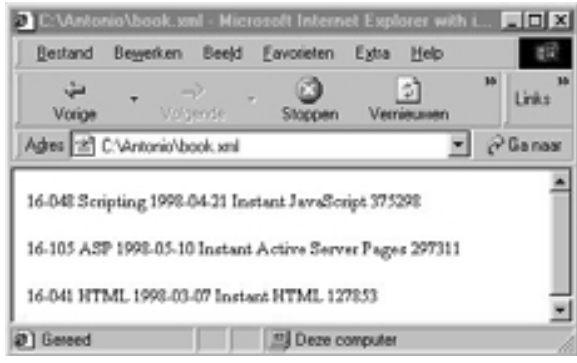

Figura 7

se ha incrementado el espacio entre elementos <libro $>$ al fijar la propiedad margin en 15 píxeles.

$<$ codigo $>,<$ categoria $>,<$ fecha_pub $>\mathrm{y}<$ titulo $>$ presentan la propiedad display:inline, por lo que se presentan en la misma línea, algo similar a lo que se obtiene usando el elemento html < span $>$. Cada uno de ellos, sin embargo, posee diferentes propiedades de estilo y por eso aparecerán en diferentes tamaños y colores. Por otra parte, no se quiere presentar el contenido de los elementos <ventas $>$ y por eso la propiedad display: para los cuales se fijó en none. La imagen de la figura 3 muestra el resultado de la hoja de estilo listadelibros.css actuando sobre el documento book.xml:

\section{Xs/t}

En este apartado se cambia de tema: de CSS a xslt; de hojas de estilo para la presentación del documento fuente a otras para su transformación. Si se quisiera analizar $x$ slt desde un punto de vista global, sería un tema demasiado amplio para un trabajo como éste. Por lo tanto, esta sección se centrará en su rendimiento práctico en el navegador Internet explorer 5 . Lo que se pretende es, por un lado, ver cómo funciona en la práctica esta tecnología y tener muy en cuenta que uno de los posibles entornos de aplicación es una herramienta tan asequible y globalmente difundida como IE5; por otro, llegar a ciertas conclusiones que nos ayudarán a la hora de plantear posibles soluciones en el mundo de la documentación digital.

\section{Introducción.}

a. Transformar código $\mathrm{xml}$ para su presentación. La función de xslt se describe a menudo como: "transformar xml para su presentación". Lo que esto realmente significa es que no hay una forma normalizada de presentar elementos xml, ya que no poseen ningún significado intrínseco.

Lo que hace $x$ slt es permitir que el autor, o el receptor del documento xml, puedan especificar cómo se debe presentar su contenido. Esto lo consigue al transformarlo en html o en un lenguaje equivalente que posea características de presentación normalizadas ${ }^{6}$. Generalmente los elementos xml se convertirán a html; en otras palabras, se especificará código html para ca- da elemento xml que será el encargado de producir la presentación deseada. Si los elementos creados son válidos en html y xml, el navegador los mostrará en pantalla. La necesidad de que todo el código cumpla con la sintaxis xml, sin dejar de ser html válido, hace que los elementos vacíos como $<h r>$ deban presentar su propio cierre $(<h r></ h r>$ o $<h r />)$. También es posible incluir secciones $<$ script $>$ en el código que serán presentadas como html y después ejecutadas por el navegador.

Lo más importante es la posibilidad de usar xslt para crear nuevos elementos o eliminar otros incluidos en el documento fuente. Esto significa que $x$ slt no está limitado al modelo CSS de aplicación de estilo a elementos existentes. Xslt presenta la posibilidad de copiar elementos desde documento fuente al nuevo, usar estructuras de decisión para presentar sólo ciertos elementos preseleccionados, crear nuevos u ordenar el contenido del documento fuente de forma diferente a la original antes de presentarlo.

b. ¿Qué hace $x$ slt? El principio básico de xslt en IE5 es que actúa como un "procesador" para transformar cualquier documento $\mathrm{xml}^{7}$ en otro diferente. Sin embargo, las hojas de estilo xslt están escritas en xml como cualquier otro tipo de documento. Por lo tanto, la entrada de datos del proceso xslt puede ser perfectamente una hoja de estilo xslt en lugar de un documento $\mathrm{xml}$ convencional ${ }^{8}$, según se ve en la figura 12 .

El resultado del proceso es otro documento, pero éste podría asumir cualquier formato. La forma en que $x s l t$ procesa los datos permite que un nodo xml pueda ser transformado en casi cualquier tipo de cadena de texto, incluyendo ascii. La gran ventaja de este planteamiento es que proporciona una técnica automática de conversión entre diferentes tipos de datos o diferentes formatos.

¿Qué ocurre cuando se lee un documento xml con IE5? Lo que se obtiene en pantalla es el resultado del trabajo de una hoja de estilo xslt. IE5 contiene una que se utiliza por defecto cuando se carga un documento xml que no especifica otra hoja. Su propósito es mostrar el documento de una forma más legible y obvia (desde la perspectiva $\mathrm{xml}$ ). Se podría decir que IE5

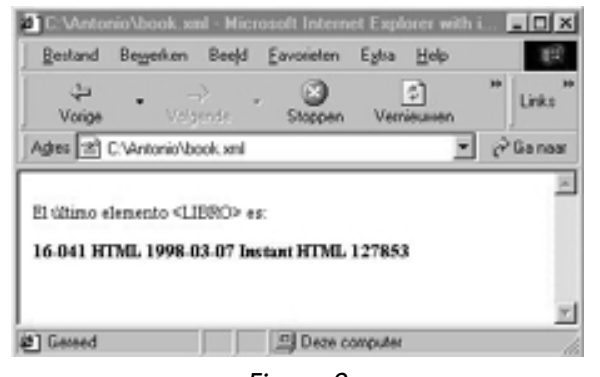

Figura 8 
"entiende" xml puesto que añade saltos de línea, indentaciones y signos $+\mathrm{y}-$ delante de los nodos con subelementos o los que se encuentran vacíos. Lo que ocurre es que IE5 está aplicando su hoja de estilo por defecto al documento xml para producir un resultado como el que se ve en la imagen de la figura 4.

c. ¿Cómo se trabaja con $x$ sit ? En términos generales, CSS relaciona la información sobre el estilo con los elementos donde lo va a aplicar especificando el nombre de estos elementos (como $\langle p>$ o $\langle e m>$ ) o usando una clase previamente definida (como .miestilo) que se aplica a un elemento utilizando su atributo class.

Xslt es completamente diferente. Usa instrucciones de proceso especiales llamadas templates para especificar los nodos-objeto ${ }^{9}$ a los que se les aplicará un conjunto de instrucciones de estilo. Los templates declaran un patrón o un filtro que pueden asociar con mucha precisión la información de estilo que contienen con elementos o grupos de ellos específicos. Los elementos-objeto no deben tener el mismo nombre o contener atributos especiales. Hay muchas formas de especificar un patrón, de forma que se seleccionen sólo los apropiados en el documento fuente $\mathrm{xml}$.

d. Xslt en el futuro. Aunque es imposible estar absolutamente seguro de lo que va a ocurrir con $x s l t$, las recomendaciones actuales del $W 3 C$ dan valiosas pistas al respecto. Actualmente las transformaciones realizadas con esta tecnología son sobre todo xml en html para que el contenido pueda ser presentado en el navegador. Hay planes para extender sus posibilidades y así permitir conversiones a otros lenguajes, particularmente aquellos mejor adaptados a funciones como resultado impreso, hablado, etc.

El borrador del $W 3 C$ para $x$ slt también propone técnicas para dar formato a la información usando "objetos de formato" o de "flujo" y un vocabulario de formato. Estos temas están en constante evolución, pero probablemente resultarán en una nueva especificación xslfo (extensible style sheets: formatting) que se ocupará del estilo en profundidad mientras xslt se concentra en las transformaciones del contenido.

2. Enlazar hojas de estilo xslt con documentos xml. Una hoja de estilo xslt es simplemente un archivo de texto, igual que una del tipo CSS. Se puede vincular un documento xml con una xslt usando una instrucción de proceso de forma similar a lo que se hizo anteriormente para unirlo con una CSS:

$<$ ?xml-stylesheet type="text/xsl" href="miestilo.xsl"?>

Sólo es posible asociar una hoja de estilo xslt a un documento; si se definen más de una, sólo la primera será interpretada y ejecutada. Si se especifican dos ho-

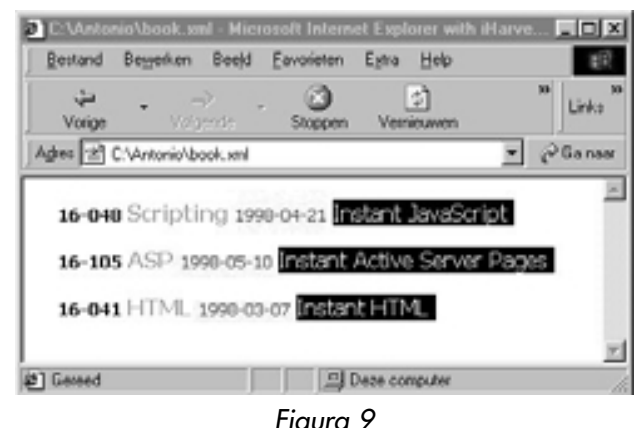

jas de estilo, una xslt y otra CSS, IE5 elegirá el primer formato. Otra circunstancia a tener en cuenta es que las hojas de estilo xslt sólo pueden cargarse desde el mismo dominio del documento xml al que están asociadas. Esto es así por motivos de seguridad, ya que pueden contener código script.

3. Templates xslt. Como ya se ha dicho, una hoja de estilo xslt se compone de una o más instrucciones especiales llamados templates. Cada uno especifica un patrón (o un filtro) que se utiliza para emparejar el template con el/los nodo/s objeto sobre los que actuará. También contienen información sobre cómo deben ser presentados.

a. Una hoja de estilo $x$ slt. Todas las instrucciones en una hoja de estilo xslt están escritas en sintaxis xml y se podrían considerar "elementos" xslt. En realidad son elementos xml ordinarios con el prefijo "xsl.". El namespace $e^{10}$ definido para ese prefijo asigna significados especiales a cada uno de esos elementos. El siguiente código muestra una hoja de estilo xslt sencilla que contiene un solo template. Obsérvese que el $n a-$ mespace debe especificarse tal y como aparece en el ejemplo para que pueda ser procesado por IE5:

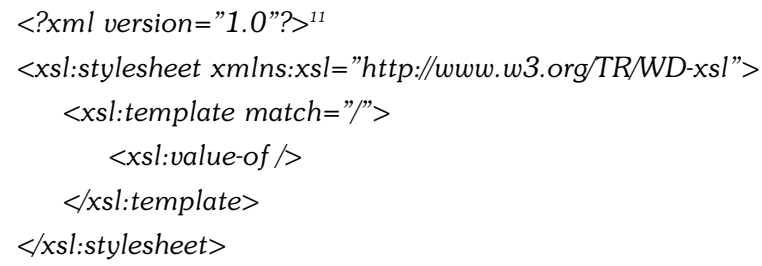

El código anterior presentará el texto del documento fuente book.xml exactamente como está, con el tipo de letra por defecto del navegador. El código tiene un elemento raíz: <xsl:stylesheet $>$, y dentro de él un sólo elemento template: $<x s l: t e m p l a t e>$, que especifica un atributo de asociación "match" con el patrón "/" como valor, lo que significa que ese template se emparejará con el elemento raíz de book.xml. Finalmente, el contenido del template (la acción) es la instrucción $x$ slt $<x$ sl:value-of $>$. Este elemento le dice al procesador: "considera como texto el contenido del nodo objeto y sus descendientes e insértalo en el documento resultado". Lo que se obtiene puede verse en la figura 5 . 
La clave del funcionamiento de las hojas de estilo $x$ slt es la forma en que los templates se asocian con los elementos del documento fuente $\mathrm{xml}$ a través del patrón especificado en el atributo "match=" del elemento $<x$ sl:template $>$. Xslt utiliza la recursión para aplicar a los elementos-objeto las instrucciones de estilo o transformación especificadas dentro de <xsl:template> (en este caso la instrucción es simplemente $<x$ sl:value-of $/>$ ).

El ejemplo anterior usaba el elemento $<x s l$ :template $>$ :

$<x s l:$ template match="/>

Aquí se especifica el patrón "/" mediante el cual se selecciona el nodo raíz del documento fuente xml. De modo que este template se emparejará unívocamente con ese elemento. La instrucción dentro del template era el siguiente elemento xslt:

$<x$ sl:value-of $>$

Esto provoca que el procesador xslt inserte en el resultado tan sólo el texto del nodo seleccionado y sus descendientes. En este caso es toda la cadena de caracteres del contenido del documento fuente, puesto que el nodo raíz contiene al resto de los nodos. Se pueden especificar diferentes templates para cada tipo de nodo usando la instrucción <xsl: apply-templates $>$ con los patrones adecuados:

vos templates para relacionarlos con los elementos hijo de los elementos anteriores y así sucesivamente, llegando a todos los niveles de profundidad del árbol documental.

\section{«Es siempre recomendable usar hojas de estilo externas por dos motivos fundamentales: pueden modificarse sin tocar el docu- mento fuente html y es posible compartirlas entre diferentes documentos»}

En la hoja de estilo anterior un solo template se asociaba con el nodo raíz del documento fuente $\mathrm{xml}^{12}$, y la acción consistía en aplicarlo (y las instrucciones anidadas en él) a los elementos descendientes del nodo raíz. Mediante la recursión, los templates de la hoja de estilo se asocian a diferentes elementos del documento fuente $\mathrm{xml}$, comenzando por el nivel superior (el elemento raíz) y recorriendo el árbol del documento hacia abajo hasta localizar todos los elementos seleccionados. Para ejemplificar este funcionamiento se puede añadir un nuevo template a la hoja de estilo anterior:

$<$ ?xml version $=$ "1.0"? $>$

$<x s l: s t y l e s h e e t x m l n s: x s l=" h t t p: / / w w w . w 3 . o r g / T R / W D-x s l ">$ $<x$ sl:template match $="$ "> $>$ $<$ xsl:apply-templates select="//libro" /> $<$ xsl:template $>$ $<x$ sl:template match $=" l i b r o ">$ $\langle p\rangle\langle$ xsl:value-of $|>\langle p\rangle$ $</$ xsl:template $>$ $</ x s l:$ stylesheet $>$

A List of Books for Wisdom

\begin{tabular}{|c|c|c|}
\hline ASP & "Instant Active Server Pages" & $\begin{array}{l}\text { Codk: } 16-105 \\
\text { Das: } 1990-05-10\end{array}$ \\
\hline HTML & "Instant HTML" & $\begin{array}{l}\text { Code: 16-041 } \\
\text { D.e: } 1990-09-07\end{array}$ \\
\hline Scripting & "Instant JavaScript" & $\begin{array}{l}\text { Code: } 16-040 \\
\text { Das:1990-04-21 }\end{array}$ \\
\hline 2000 Whotom Sorte. & & \\
\hline
\end{tabular}

Figura 10

$<x$ sl:apply-templates select="patrón"/>

La forma más simple de seleccionar un nodo es referenciándolo por su nombre - lo que asegura que la acción se llevará a cabo sobre todos los que tengan ese nombre-, lo cual, en la mayoría de los casos, es lo que se pretende conseguir. Sin embargo, la técnica mencionada (<xsl: apply-templates $>)$ permite asociar templates a los elementos hijo de la raíz, seleccionándolos mediante el patrón, especificado en el atributo "select". Después, sucesivamente, se pueden añadir más instrucciones $<x s l$ : apply-templates $>$ a esos nue-
Lo que se ha hecho es sustituir el elemento $<x$ sl:value-of $>$ en el template original con $<x$ sl:apply-templates select="//libro" $>$, que comunica al procesador xslt que debe aplicar el template asociado con el patrón //libro a cada elemento <libro $>$ en el documento fuente $\mathrm{xml}$ (el patrón //libro se asocia con todos <libro $>$ a cualquier nivel por debajo del nodo raíz). El nuevo template que se ha añadido a la hoja de estilo se asocia con //libro y las instrucciones anidadas dentro de él se ejecutarán para cada $<$ libro $>$. Quizás se ve un poco más claro en la imagen de la figura 6 .

En el template rodeado por la línea roja se usa de nuevo el elemento $<x$ sl:value-of $>$ para dar formato al resultado de cada elemento $\langle$ libro $\rangle$. Se puede observar que, en este caso, se rodea el elemento $<x s l$ :valueof $>$ con la etiqueta html $\langle p\rangle\langle/ p\rangle$, con lo que cada $<$ libro $>$ (y sus descendientes ${ }^{13}$ ) aparecerá en un párrafo aparte. En la imagen de la figura 7 se puede apreciar el resultado. 
b. Sintaxis IE5-xslt de asociación de patrones. $\mathrm{El}$ atributo "match" del elemento <xsl:template $>$ proporciona varias posibilidades a la hora de especificar exactamente qué nodos (elementos o atributos) del documento fuente $\mathrm{xml}$ deben ser procesados por un template particular. La primera hoja de estilo usaba "/" para referirse al nodo raíz, pero hay muchas más posibilidades. Básicamente hay dos formas de especificar nodos:

- Mediante la posición del nodo o grupo de nodos en el árbol jerárquico del documento fuente.

- A través de la aplicación de un filtro que seleccione uno o varios nodos.

b.1. Operadores de posición para xslt e IE5. Para seleccionar un nodo o nodos usando su posición en el árbol del documento fuente se usa un conjunto de operadores de posición o ruta; combinándolos se obtiene un patrón que, como se ha visto, se

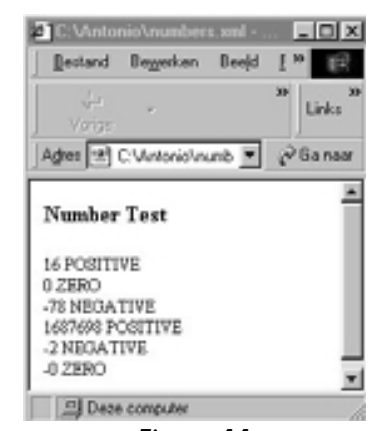

Figura 11 listadelibros/libro/titulo[end()]

obtendríamos el último elemento $<$ titulo $>$ del primer $<$ libro $>$.

b.2. Operadores de filtro para xslt e IE5. Un filtro xslt tiene la forma: [operador patrón], donde opera$d o r$ es un filtro opcional que define cómo se va a aplicar el patrón y patrón, es el filtro xslt que selecciona uno o varios elementos en el documento fuente basándose en diferentes criterios. Es posible elegir elementos basándose en:

— La existencia de elementos hijos.

- El valor de un elemento o de un elemento hijo.

— La presencia de atributos.

— El valor de éstos.

- Cualquier combinación de las posibilidades anteriores.

El siguiente código, por ejemplo, usa el filtro xslt [titulo] para seleccionar solamente los elementos $<$ categoria $\rangle$ de libros que tengan elementos hijo $<$ titulo $>$ (por lo tanto no se seleccionarán elementos $<$ libro $>$ sin hijos $<$ titulo $>$ ):

libroltituloycategoria

Para encontrar todos los libros que tengan hijos $<$ titulo $>$ y $<$ categoria $>$ se usarán dos filtros:

libro[titulo][categoria]

Para seleccionar un elemento por su valor (por ejemplo, elementos <libro> que contengan un elemento <categoria $>$ cuyo valor sea "Scripting") se podría usar el siguiente código:

libro[categoria $=$ 'Scripting']

Hay, además del =, varios operadores de comparación que pueden usarse:

- libro[ventas $>10000]$.

— libro[fecha_pub <= '1998-02-07'].

— libro[titulo! = 'Instant JavaScript'].

— libro[titulo \$ine\$ 'Instant JavaScript'].

El último ejemplo usa un operador de comparación "no igual a" insensible a mayúsculas o minúsculas. Es diferente del anterior, que sí tiene en cuenta esa diferencia. Un filtro puede usar también el operador @ para especificar un atributo del elemento actual. El siguiente, por ejemplo, especifica que el elemento $<l i$ bro $>$ debe tener un atributo fecha_impr:

libro[@fecha_impr]

Cuando un filtro especifica el valor de un atributo, puede usar * para definir cualquier nombre de atribu- 
to. Con este, por ejemplo, sólo se seleccionan elementos <autor > con un atributo tipo cuyo valor sea "contrato":

, autor[@tipo='contrato']

El siguiente filtro obtiene elementos <autor $>$ en los que cualquiera de sus atributos tenga un valor "contrato":

autor[@* = contrato']

Finalmente, es posible construir filtros más complejos usando operadores booleanos para combinar otros simples. Los disponibles son: \$and\$, \$or\$ y $\$$ not\$. En el siguiente código se seleccionan todos los elementos <libro > que tengan un elemento hijo <categoria> con el valor 'Scripting' pero no uno con el valor ' $h t m l$ ':

libro[categoria = 'Scripting' \$and $\$$ categoria != 'html']

libro[categoria = 'Scripting' \$and\$ \$not $\$$ categoria $=$ ' $h$ tml']

b.3. Coincidencia de varios patrones con un sólo elemento. Si hay más de un template en una hoja de estilo, es fácil que algunos elementos del documento fuente estén asociados a más de un patrón en los templates. Para impedir la duplicación en el resultado, el procesador xslt no permitirá que se aplique más de un template a cada elemento y decide cuál debe usarse comprobando todos los que tiene asociados y eligiendo el que mejor se corresponda con él. Sin embargo, es mejor evitar estas situaciones. Un ejemplo: un elemento <autor $>$ hijo de un elemento <libro>, podría relacionarse a varios templates diferentes: "libro/autor", "libro/*” o “*”. En este caso el procesador escogería el primero porque se trata de la a s ociación más restrictiva, es decir, la que mejor se corresponde con el ele$\mathrm{m}$ e $\mathrm{n}$ t $\mathrm{o}$ $<$ autor $>$.

\section{Ele-}

$$
\begin{aligned}
& \text { — }<x s l: \text { copy }>\text {. } \\
& \text { — }<x \text { sl:value-of }>\text {. }
\end{aligned}
$$
xsl. La sintaxis es:

$</ x s l: s t y l e s h e e t>$ mento resultado. sintaxis es: $</ x s l:$ template $>$

mentos $x$ slt

1. <xsl:stylesheet $>$. Es el elemento raíz de una hoja de estilo xslt y, por lo tanto, contiene a los demás elementos. En él se puede especificar el lenguaje de script usado en la hoja, si se utiliza alguno, y si necesitan preservarse o no los espacios blancos del documento fuente en el que se da como resultado. Además contiene una declaración de namespace para el prefijo

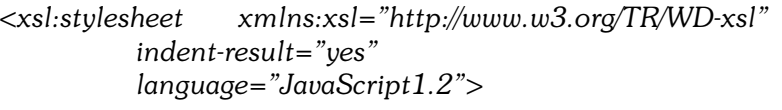

El namespace (xmlns) debe teclearse exactamente como en el ejemplo para que pueda ser leído por IE5. $\mathrm{El}$ atributo "indent-result" con valor positivo hace que los espacios en blanco sean preservados en el docu-

2. <xsl:template>. Define un template específico dentro de una hoja de estilo xslt. Utiliza el atributo "match" cuyo valor es un patrón de asociación dado para especificar los elementos en el documento fuente xml que serán seleccionados y procesados por la hoja de estilo. También acepta un atributo para especificar el lenguaje script que se va a usar en el template. La

$<x s$ : template match="patrón" language="VBScript">

Documento $\mathrm{xml}$
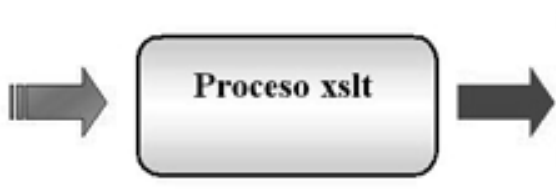

Hoja de estilo xslt

Documento html

Otros formatos

Figura 12

$$
\text { Hoja de estilo xslt }
$$

Documento $\mathrm{xml}$

3. <xsl:define-templateset $>$. Este elemento se usa para agrupar un conjunto de templates que tienen un ámbito diferente al del resto de la hoja de estilo. Puede usarse dentro básicos. IE5

soporta varios elementos o instrucciones xslt que pueden usarse en una hoja de estilo xslt. Los fundamentales son:

$$
\begin{aligned}
& \text { — }<x \text { sl:stylesheet }>. \\
& -<x \text { sl:template }>. \\
& -<x \text { sl:define-template-set }>. \\
& -<x s l: \text { apply-templates }>.
\end{aligned}
$$

de los elementos <xsl:stylesheet $>$ o $<x$ sl:template $>$. El siguiente código define dos templates que sólo se aplicarán cuando se use el elemento (<xsl:template match $=$ "*” $>$ ) en el que se hallan anidadas:

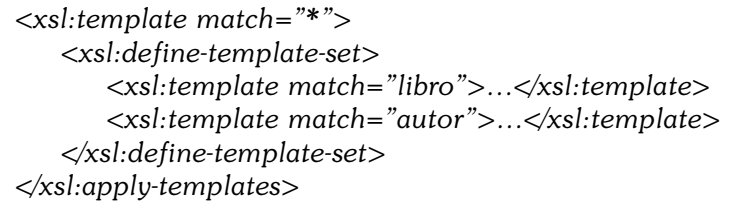




\begin{tabular}{|c|c|}
\hline Operador & Descripción \\
\hline I & $\begin{array}{l}\text { Es el operador de posición que identifica a la descendencia. Selecciona elementos que son } \\
\text { descendientes directos del nodo especificado. Es similar al sistema usado para especificar } \\
\text { direcciones en un URL, por ejemplo: libro/categoria elige todos los elementos <categoria> } \\
\text { descendientes de বlibro>. Para definir el nodo raíz se sitúa este operador al principio del } \\
\text { patrón de la siguiente forma: /libro/categoría. }\end{array}$ \\
\hline$/ /$ & $\begin{array}{l}\text { Indica descendencia recursiva. Selecciona todos los nodos especificados a cualquier nivel de } \\
\text { profundidad bajo el nodo corriente. Por lo tanto listadelibros//titulo seleccionaría todos los } \\
\text { elementos «titulo }>\text { que sean descendientes a cualquier nivel del elemento <listadelibros }>\text {. } \\
\text { Cuando este operador aparece al comienzo del patrón: //titulo obtiene todos los elementos } \\
\langle\text { titulo }>\text { que sean descendientes del nodo raíz (por lo tanto todos los elementos }\langle\text { titulo } \gg \text { ). }\end{array}$ \\
\hline . & $\begin{array}{l}\text { Es el operador de contexto corriente. Se usa para indicar el nodo corriente o "contexto". De } \\
\text { esta forma //titulo seleccionará todos los elementos <titulo> a cualquier nivel por debajo del } \\
\text { nodo corriente. La combinación. / siempre indica el contexto actual y normalmente es } \\
\text { superflua: ./libro/categoria es lo mismo que libro/categoria. }\end{array}$ \\
\hline (a) & $\begin{array}{l}\text { Identifica la posición de los atributos. Indica que esa sección del patrón se está refiriendo a } \\
\text { atributos del elemento corriente. Así libro@fecha_impr seleccionará el valor del atributo } \\
\text { "fecha_impr" para cada uno de los elementos বlibros. }\end{array}$ \\
\hline * & $\begin{array}{l}\text { El asterisco es un operador comodín que se usa cuando se desean seleccionar todos los } \\
\text { elementos o atributos sin tener en cuenta sus nombres. Por ejemplo libro/* seleccionara todos } \\
\text { los hijos de todos los elementos বlibros, libro/@* obtendrá todos los atributos de esos } \\
\text { mismos elementos. }\end{array}$ \\
\hline
\end{tabular}

Tabla 1

$</ x s l:$ template $>$

4. <xsl:apply-templates $>$. Este elemento le indica al procesador que debe buscar y ejecutar los templates asociados a los elementos hijos del elemento actual. Si hay un atributo "select" cuyo valor sea un patrón de asociación, los elementos seleccionados según ese patrón serán los que se procesen. También acepta el atributo "order-by" que determina el orden en que se presentarán dichos elementos. El valor de este atributo es una lista de patrones separados por ";” cada uno de los cuales puede ser precedido por "+" o "-" para indicar orden ascendente o descendente. Los patrones en la lista se procesarán en relación con aquel dado en el atributo "select". La sintaxis es: nes"/>

$<x s l:$ apply-templates select="patrón" order-by="lista de patro-

Para confeccionar una lista de libros ordenados alfabéticamente por el nombre del autor, se usaría el siguiente código:

$$
\begin{array}{r}
<x s l: a p p l y-t e m p l a t e s \quad \text { select="libro" } \\
\text { order-by="+autor/apellido; +autor/nombredepila"> }
\end{array}
$$

5. $<x$ sl: $\operatorname{copy}^{14}>$. Simplemente copia los elementos seleccionados al resultado como están, es decir, incluyendo sus etiquetas. Ésta es la diferencia respecto de $<x s l$ :value-of..> que sólo copiaba el contenido de los elementos elegidos:

$$
\begin{aligned}
& <x \text { sl:template match }=" * "> \\
& \quad<x s l: \text { copy }> \\
& <\mid x \text { xsl:template }>
\end{aligned}
$$

6. $<x$ sl:value-of $>$. Devuelve el valor de un nodo $\mathrm{xml}$ (y de sus descendientes si los tiene) en forma de texto que se inserta en el resultado. Se puede usar op- cionalmente un patrón (como valor del atributo "select") para seleccionar uno o varios elementos en el documento fuente. Si se omite el atributo "select" se optará por el elemento actual en el documento fuente. Si el patrón se asocia con más de un elemento, sólo se procesará el primero de ellos. $\mathrm{Si}$ el elemento tiene descendientes, sus valores se concatenarán en el resultado. La sintaxis es: trón" $>$

$<x s l: v a l u e-o f$ select="pa-

e. Generar html mediante $\boldsymbol{x}$ slt. Los elementos $x$ slt que se han comentado hasta ahora son suficientes para construir hojas de estilo de una complejidad razonable y con capacidad semejante a las construidas con CSS. Se ha visto cómo xslt procesa los documentos xml emparejando los templates de la hoja de estilo con los elementos del documento fuente y generando a partir de ahí un documento resultante en el que los elementos originales han sido simplemente copiados o bien procesados hasta cierto punto. La mayoría de los ejemplos anteriores generaban como resultado código html que podía ser gestionado por el navegador de la forma usual.

Para ver mejor cómo puede usarse xslt para dar formato a un documento xml, en los siguientes ejemplos se va a definir una hoja de estilo xslt que, asociada al archivo book.xml, producirá el mismo resultado que la hoja de estilo CSS que se usó al principio. En la primera sección de la hoja de estilo xslt se puede ver que el template raíz (cuyo atributo "match" tiene el valor “/”) contiene código html con el que se creará la estructura básica del documento resultante. Dentro del html hay un elemento $<x$ sl:apply-templates...> que selecciona los elementos <libro $>$ del documento fuente:

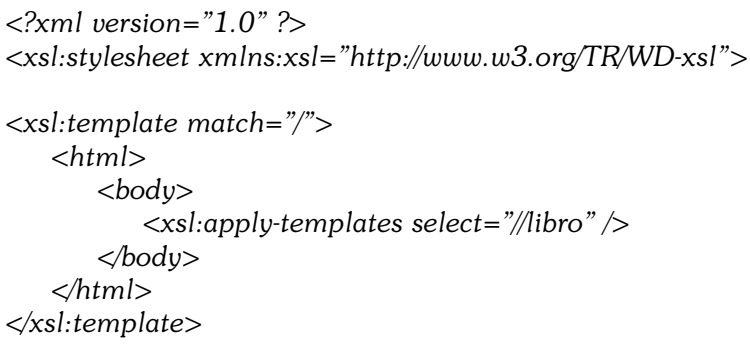

La hoja de estilo anterior transformará book.xml en el siguiente documento html: 


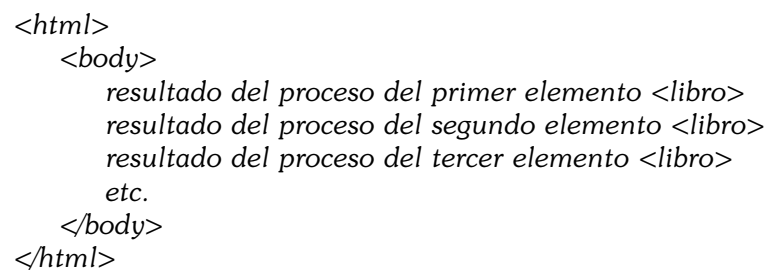

Al procesar el template raíz, el procesador xslt encuentra el patrón "//libro" y busca todos los elementos $<$ libro > bajo la raíz en el documento fuente. Al encontrarlos, busca en la hoja de estilo un template que se asocie con ellos, como el siguiente:

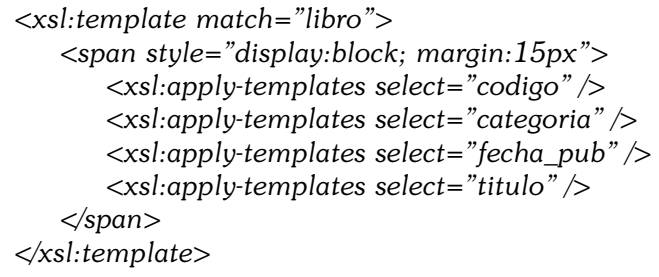

Este template simplemente inserta un elemento html <span> para cada elemento <libro $>$, dentro del cual se incluyen las referencias a los templates asignados a cada uno de los elementos hijos de <libro>: $<$ codigo $>,<$ categoria $>$, etc.

Las propiedades de estilo usadas con el elemento $<$ span> son exactamente las mismas que se utilizaron en la hoja de estilo CSS. El resultado es simplemente que el contenido de cada elemento <libro> aparecerá en un bloque aparte, dado que se ha vuelto a usar la propiedad display:block. Lo único que falta para completar la hoja de estilo xslt es, por lo tanto, añadir el formato que deseemos a los templates asignados a los elementos: <codigo $>,<$ categoria $\rangle,\langle$ fecha pub $>$ y $<$ titulo $>$. Para $<$ categoria $>$, por ejemplo, podría ser el siguiente:

$<x$ sl:template match ="categoria" > $<$ span style $=$

display:inline;

font-family:Ta-

homa, Arial,sans-serif;

font-size: $12 p t$ color:darkgray;

weight:bold"> $</$ span $>$

$</ x$ sl:template $>$

De nuevo, es un simple elemento $<$ span > dentro del cual se inserta el contenido del elemento <categoria> (en el documento fuente book.xml) mediante el uso de la instrucción <xsl:value$o f . .>$. El estilo especificado es idéntico al que se usó en la hoja de estilo CSS. Los otros tres templates, para los elementos $\langle$ codigo $\rangle,\langle$ fecha pub $\rangle$ y $<$ titulo $>$, siguen exactamente el mismo criterio. La hoja de estilo xslt completa es:

$<$ ?xml version $=$ "1.0"? $>$

$<x s l:$ stylesheet $x m l n s: x s l=" h t t p: / / w w w . w 3 . o r g / t r / w d-x s l ">$

$<x$ sl:template match $="$ ">

$<$ html $>$

$<$ body $>$

$<x$ sl:apply-templates select="//libro" />

$</$ body $>$

$<$ html $>$

$</$ xsl:template $>$

$<$ xsl:template match="libro">

<span style="display:block; margin:15px">

$<x$ sl:apply-templates select="codigo" $>$

$<x$ sl:apply-templates select="categoria" $>$

$<x$ sl:apply-templates select="fecha_pub" />

$<x$ sl:apply-templates select="titulo" $>$

$</$ span $>$

$</$ xsl:template $>$

$<x s l$ template match $=$ "codigo" $>$

$<$ span style =" display:inline;

font-family:tahoma, arial,sans-serif;

font-size:10pt;

font-weight:bold">

$<x$ sl:value-of $1>$

$</$ span $>$

$</$ xsl:template $>$

$<x$ sl:template match="categoria">

$<$ span style=" display:inline;

font-family:tahoma, arial,sans-serif;

color:darkgray; font-size:12pt;

font-weight:bold">

$<$ xsl:value-of $/>$

$</$ span $>$

$<\mid x s l:$ template $>$

$<x$ sl:template match="fecha_pub">

$<$ span style=" display:inline;

font-family:tahoma, arial,sans-serif;

color:red;

font-size:10pt">

\begin{tabular}{|c|c|}
\hline Patrón & Resultado \\
\hline / & Solamente el nodo raiz. \\
\hline libro/autor & Elementos $<$ autor $>$ hijos de elementos $<$ libro $>$. \\
\hline // & El nodo raíz y todos los nodos bajo el. \\
\hline$/ /{ }^{*}$ & Todos los elementos bajo el nodo raiz. \\
\hline libro//autor & Elementos $\langle$ autor $>$ descendientes a cualquier nivel de elementos $\langle$ libro $\rangle$. \\
\hline //autor & Elementos < autor $>$ descendientes a cualquier nivel del elemento actual. \\
\hline * & Todos los elementos excepto el nodo raíz. \\
\hline libro/* & Todos los elementos hijos de $\langle$ libro $>$. \\
\hline libro//* & Todos los elementos descendientes a cualquier nivel de $\langle$ libro $\rangle$. \\
\hline libro/*/autor & Elementos < autor $>$ nietos de elementos $<$ libro $>$. \\
\hline libro/@fecha_impr & Atributos "fecha_impr" asociados a elementos $\langle$ libro $\rangle$. \\
\hline */@fecha_impr & Atributos "fecha_impr" asociados a cualquier elemento. \\
\hline
\end{tabular}

Tabla 2 


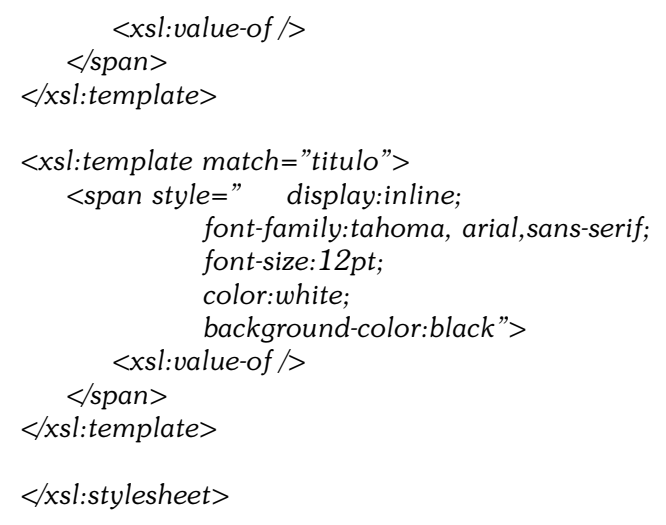

Y el resultado en el navegador es idéntico al conseguido con CSS (figura 9).

Una diferencia sutil que no puede apreciarse en el ejemplo es que con CSS los elementos se presentan en el orden en que están dispuestos dentro del documento fuente xml, mientras que con xslt aparecen según están dispuestos en los elementos <xsl:apply-templates...> en la hoja de estilo xslt. Después de haber comprobado que $x s l t$ es capaz de llevar a cabo todo lo que CSS puede hacer, sería interesante ver si también es posible realizar otras que CSS no puede realizar. La forma más coherente de comprobarlo es llevando el ejemplo anterior un poco más lejos. La imagen de la figura 10 es el resultado de aplicar una nueva hoja de estilo xslt al mismo documento book.xml. Es fácil apreciar que se trata de un resultado completamente diferente.

\section{«El principio básico de xs/t en IE5 es que actúa como un 'pro- cesador' para transformar cual- quier documento $\mathrm{xml}$ en otro di- ferente»}

Aunque parezca muy distinto, básicamente sigue tratándose de la transformación de un documento xml en html. Sin embargo se usan algunos trucos como añadir nuevos elementos al resultado o cambiar el orden de presentación de los elementos xml originales. Ahora $<$ categoria $>$ aparece en primer lugar seguido de $<$ titulo $>$. Esto podría haberse hecho usando las propiedades de posicionamiento absoluto en CSS, pero lo que ocurre en esta página es que los elementos están en celdas de una tabla html. Además los libros aparecen ordenados alfabéticamente. ¿Qué ha cambiado en la hoja de estilo?

d.1. La hoja de estilo del ejemplo anterior puede dividirse en tres partes.

- Primera parte:

$<$ ?xml version $=$ "1.0"? $>$

$<x s l:$ stylesheet xmlns:xsl="http://www.w3.org/TR/WD-xsl">

$<x$ sl:template match $="$ ">

$<h t m l>$

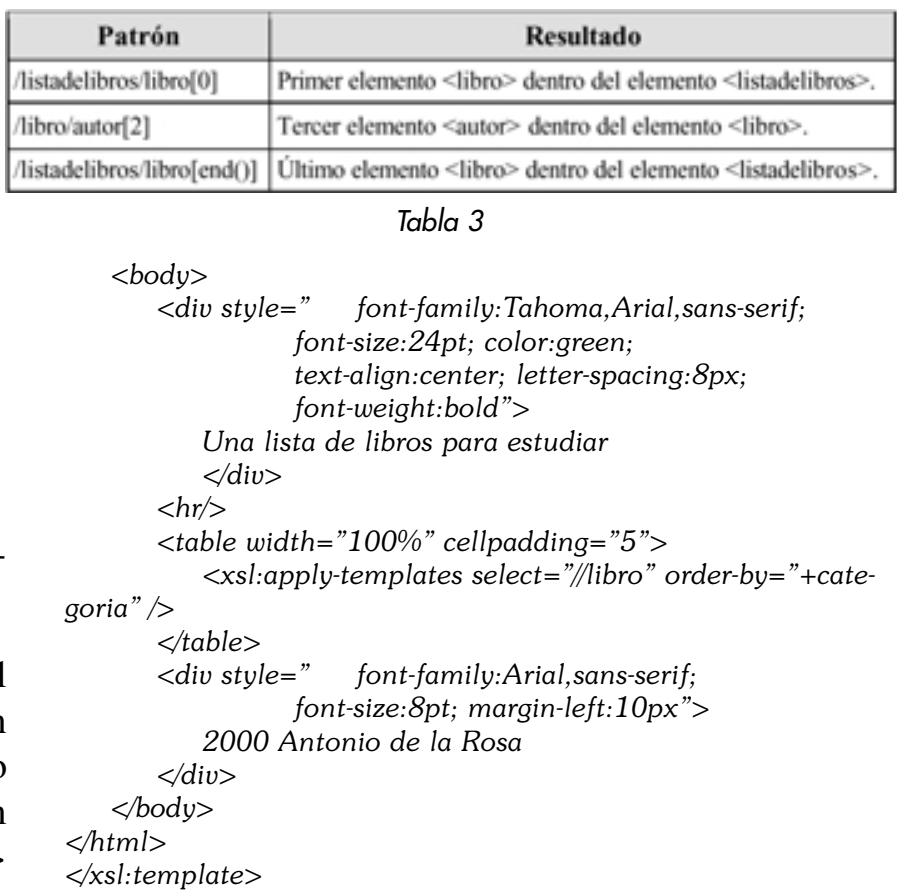

Tabla 3

$<$ body $>$

$<$ div style =" font-family:Tahoma,Arial,sans-serif; font-size:24pt; color:green; text-align:center; letter-spacing:8px; font-weight:bold">

Una lista de libros para estudiar $</$ div $>$

$<h r />$

$<$ table width $=$ "100\%" cellpadding $=" 5 ">$

$<x$ sl:apply-templates select="//libro" order-by="+categoria" $/>$

$</$ table $>$

$<$ div style=" font-family:Arial,sans-serif; font-size:8pt; margin-left:10px"> 2000 Antonio de la Rosa

$</$ div $>$

$</$ body $>$

$</$ html $>$

$<$ xsl:template $>$

Para el encabezamiento se usa un elemento html $<d i v>$ al que se aplican las propiedades de estilo apropiadas. Después una línea horizontal $\langle h r /\rangle$. A continuación se incluye una tabla donde se inserta la instrucción <xslt:apply-templates...> encargada de seleccionar los elementos $<$ libro $>$ del documento fuente. A la tabla le sigue otro elemento $<d i v>$ que contiene una nota al pie. En otras palabras, se crea una tabla html que va a contener el contenido del documento xml book.xml. Para hacer que cada elemento <libro $>$ se inserte en una fila separada en la tabla, se añade al código anterior lo siguiente.

- Segunda parte:

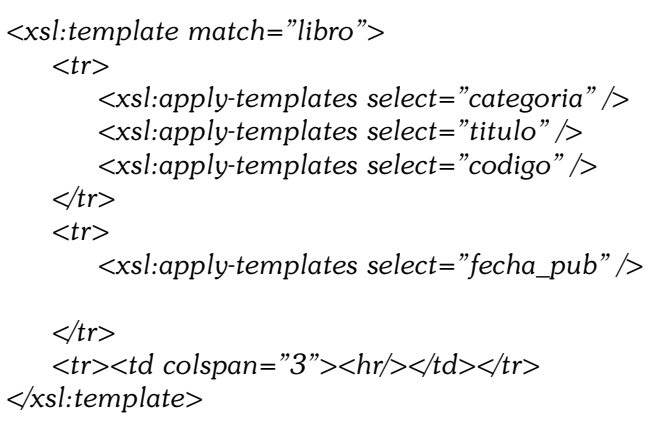

En este ejemplo se puede ver cómo se cambia en el resultado el orden de los elementos. Sólo es necesario especificar el template para cada uno según se desea que aparezcan: <categoria $>$ en primer lugar, seguido por $\langle$ titulo $\rangle,\langle$ codigo $\rangle$ y $\langle$ fecha pub $\rangle$. Este template es más complicado por el hecho de que las celdas que van a contener los elementos $<$ categoria $>$ y $<t i$ tulo $>$ deben abarcar dos columnas, mientras que la columna final contiene $<$ codigo $>$ y $<f e c h a$ p $u b\rangle$ en celdas ordinarias. Esa es la razón para emplear la etiqueta $\langle$ tr $\rangle<|t r\rangle$ como se ha hecho porque, de esa forma, se fuerza al contenido del elemento $<f e c h a$ pub $>$ a 
aparecer en la primera celda disponible dentro de la siguiente fila, es decir: debajo de la celda de $<$ codigo $>$. Entre cada elemento <libro $>$ hay otra fila que abarca las tres columnas descritas y que contiene una línea horizontal. Gráficamente se podría describir según la tabla 4 .

Para completar la hoja de estilo xslt que produce la imagen mostrada anteriormente, sólo se necesitan los cuatro templates referenciados en el código anterior y correspondientes a los elementos $<$ categoria $>,<$ titulo $>,<$ codigo $>$ y $<$ fecha pub $>$.

- Tercera parte:

$<x s l:$ template match $=$ "codigo" $>$

$<t d$ style $=" \quad$ font-family:Tahoma, Arial,sans-serif; Font-size:10pt"> $</ t d>$

Código: $\langle b><$ xsl:value-of $/></ b>$

$</ x$ sl:template $>$

$<x$ sl:template match="categoria" $>$

$<t d$ rowspan $=" 2 "$ style =" font-family:Comic Sans MS, Arial,sans-serif;

Color:darkblue; font-size:16pt;

$</ t d>$

Font-weight:bold">

$<\mid x s l:$ template $>$

$<x s l:$ template match="fecha_pub">

$<t d$ style $=" \quad$ font-family:Tahoma, Arial,sans-serif; Font-size:10pt" $>$

Fecha: $\langle b\rangle\langle x$ sl:value-of $|><| b>$

$</ t d>$

$</$ xsl:template $>$

$<x s l:$ template match $=$ "titulo">

$<t d$ rowspan="2" style ="

Font-family:Lucida Casual Italic,sans-serif;

Font-size:18pt;

Color:darkred;

Font-weight:bold">

$</ t d>$ $<i>$ " $<$ xsl:value-of $/>$ " $<i>$

$</ x$ sl:template $>$

$<\mid x s l:$ stylesheet $>$

Puede observarse que las etiquetas $\langle t d\rangle\langle/ t d\rangle$ se insertan en las posiciones adecuadas con los atributos "rowspan" correspondientes para las celdas que contienen los elementos $<$ categoria $>$ y $<$ titulo $>$. No hay necesidad de reorganizar los templates porque son ejecutados en el orden especificado según el principal (el de $<$ libro $>$ en la segunda parte). En definitiva, sólo hay que unir las tres partes comentadas en una sola hoja de estilo xslt, y ésta, asociada al documento book.xml, producirá el resultado que ya se ha visto (figura 10).

e. Crear elementos $\mathrm{xml}$ en el resultado. Con xslt se pueden crear nuevos elementos xml en el documento resultante, con lo cual se proporciona un método para mantener la documentación constantemente actualizada sin tener que cambiar, necesariamente, los documentos fuente. Los elementos xslt con los que se consigue esto son:

—Xsl:attribute.

- Xsl:cdata.

-Xsl:comment.

— Xsl:element.

—Xsl:entity-ref.

-Xsl:pi.

- Xslt también tiene un elemento que devuelve el nombre del nodo actual en forma de texto: xsl:nodename.

1. Xsl:attribute. Crea un atributo $\mathrm{xml}$ con el nombre y contenido que se especifique:

$<x$ sl:attribute name="nombre del atributo" $>$ Valor del atributo $<\mid$ xsl:attribute $>$

Por ejemplo, para añadir a todos lo elementos $<a u$ tor $>$ un atributo llamado "tipodeautor" y el valor "investigador" se usaría el siguiente código:

<xsl:template match:"/>

$<x$ sl:apply-templates select="//autor" $>$ $<\mid$ xsl:template $>$

$<x$ sl:template match:"autor"> $<x s l: c o p y>$ bute>

$<x$ sl:attribute name="tipodeautor" $>$ Investigador $<\mid x$ sl:attri-

$<\mid x s l: c o p y>$

$</$ xsl:template $>$

\begin{tabular}{|c|c|c|}
\hline \multirow{2}{*}{$<$ categoria $>$} & \multirow{2}{*}{$<$ titulo $>$} & $\mid<$ codigo $>$ \\
\hline & & $<$ fecha_pub $>$ \\
\hline \multicolumn{3}{|l|}{$\langle h r />$} \\
\hline \multirow{2}{*}{$<$ categoria $>$} & \multirow{2}{*}{$<$ titulo $>$} & $<$ codigo $>$ \\
\hline & & $<$ fecha_pub $>$ \\
\hline \multicolumn{3}{|l|}{$<h r />$} \\
\hline \multirow{2}{*}{$<$ categoria $>$} & \multirow{2}{*}{$<$ titulo $>$} & $<$ codigo $>$ \\
\hline & & $<$ fecha_pub $>$ \\
\hline$<h r />$ & & \\
\hline
\end{tabular}

Si se quisiera añadir el atributo solamente al último autor, habría que modificar el código de la siguiente forma:

$<x$ sl:template match:"">

$<x$ sl:apply-templates select="//autor" $>$ $</$ xsl:template $>$

$<x s l: t e m p l a t e ~ m a t c h: " a u-$ tor[end()]"> $<x s l: c o p y>$ $<x s l: a t t r i b u t e ~ n a-$ me="tipodeautor">investi- 
El siguiente ejemplo de hoja de estilo xslt toma el documento book.xml y crea otro nuevo a partir de él insertando una instrucción de proceso y un comentario, seleccionando solamente tres elementos del documento original. También se limitan los libros presentados usando un filtro que obtiene sólo aquellos publicados después del 31 de marzo de 1998:

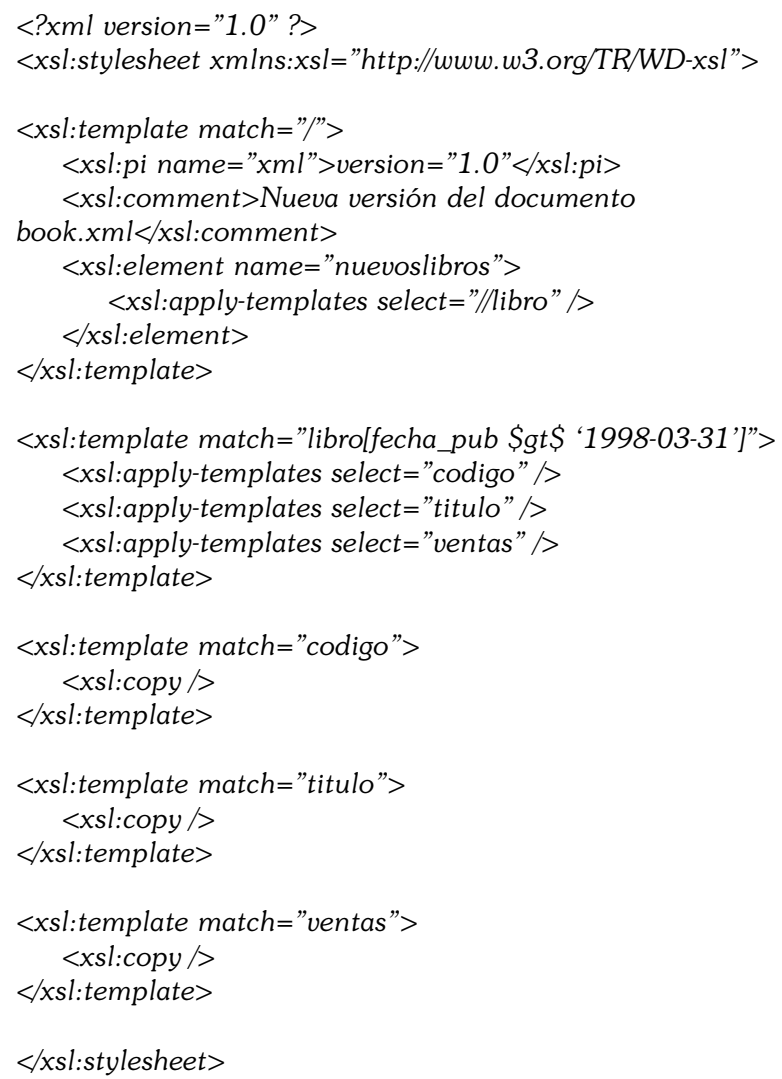

Después de seleccionar los elementos <libro $>$ que se quieren insertar en el documento resultante, se especifican los templates para los elementos hijo: $<$ codigo $>,<$ titulo $>$ y $<$ ventas $>$ que deben copiar los elementos a los que se refieren en el documento resultante usando la instrucción $\langle x s l t$ :copy $>$. El resultado de aplicar la hoja de estilo anterior al documento book.xml (a la izquierda) es un nuevo documento xml (a la derecha) con estructura y contenido diferentes (tabla 5).

\section{«La forma en que xslt procesa los datos permite que un nodo $\mathrm{xml}$ pueda ser transformado en casi cualquier tipo de cadena de texto, incluyendo asciì}

g. Programación en xslt. Además de dar formato al contenido de un documento xml o crear nuevos elementos, el procesador xslt también puede controlar el resultado usando elementos de loop y estructuras de decisión. Es parecido a la forma en que $S Q L$ puede examinar los resultados obtenidos al consultar una base de datos. Además, también se pueden incluir scripts en las hojas de estilo xslt, lo que resulta particularmente útil pues es actualmente la única técnica para incluirlos en documentos xml puros.

g.1. Loops y estructuras de decisión. Hay cinco elementos básicos en xslt que proporcionan a las hojas de estilo la capacidad de definir loops y estructuras de decisión:

$$
\begin{aligned}
& \text { —Xsl:for-each } \\
& \text { — Xsl:if } \\
& \text { —Xsl:choose } \\
& \text { - Xsl:when } \\
& \text { - Xsl:otherwise }
\end{aligned}
$$

1. Xsl:for-each. Se utiliza para detallar series de elementos xslt sobre los que se va a repetir un determinado proceso. Esta instrucción permite controlar el orden de los elementos a los que afecta sin tener que especificar templates en un orden determinado (como ocurría con el elemento <xsl:apply-templates...>). Si el atributo "select" presenta algún valor, sólo se procesarán los elementos hijos del elemento o aquellos a los que se refiera ese valor. También acepta un atributo opcional "order-by" que define el orden en que se presentarán los elementos seleccionados. El valor de este atributo es un conjunto de patrones delimitados por ",", cada uno de los cuales puede estar precedido por “+” o “- " para indicar orden ascendente o descendente. Su sintaxis general es:

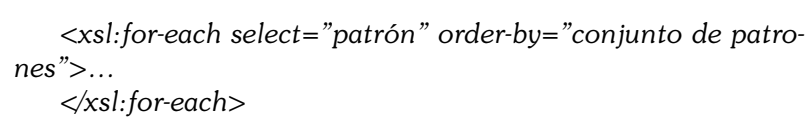

Para conseguir, a partir de book.xml una lista de nombres de autores en orden alfabético, se podría usar el siguiente código:

$<x$ l:for-each select="libro/autor" order-by="+apellido; +nombredepila"> ...

Aquí los elementos xslt que deban procesarse para cada elemento <autor $>$.

$</$ ssl:for-each $>$

2. Xsl:if. Se usa para obtener un resultado condicionado al producido por una prueba, la cual no es más que un patrón $x s l t$ :

$<x s l:$ if match="patrón" $>$ Resultado condicionado $</ x$ sl:if $>$

El siguiente código insertará el texto Investigador solamente si el atributo "tipodeautor" de <autor > presenta un valor "investigador":

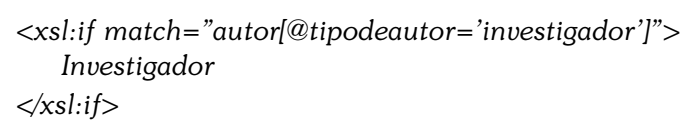

3. Xsl:choose, Xsl:when y Xsl:otherwise. Se usan conjuntamente para proporcionar al procesador $x$ slt capacidad de bifurcación condicional. $<x s l$ :when.. $>$ pre- 
senta un atributo "match", cuyo valor, como en el caso de $<x$ sl:if.. $>$, es la prueba sobre la que se construye la condición. Si el contenido del documento fuente xml supera esa prueba, entonces, el contenido de $<x$ sl:when.. $>$ se incluirá en el resultado. Solamente se procesa el primer elemento $<x$ sl:when.. $>$ que supera la condición y si no hay ninguno, se utiliza $<x$ sl:otherwise..> en su lugar. Por ejemplo, dado el documento $\mathrm{xml}$ numeros.xml:

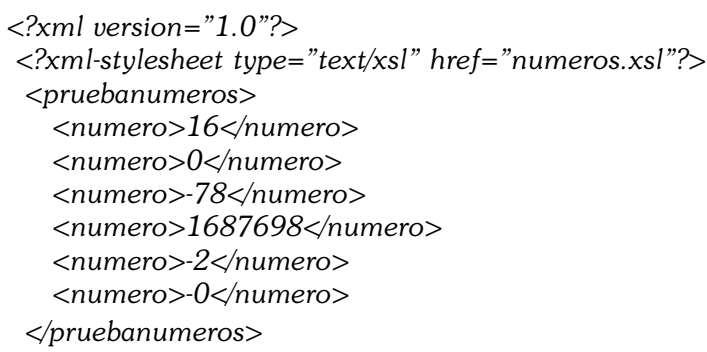

se puede crear una hoja de estilo (numeros. $x$ sl) que indique cuándo un número es positivo, negativo o cero usando los elementos $\langle x$ sl:choose $>,<x s l$ when $>$ y $<x s l$ :otherwise $>$ :

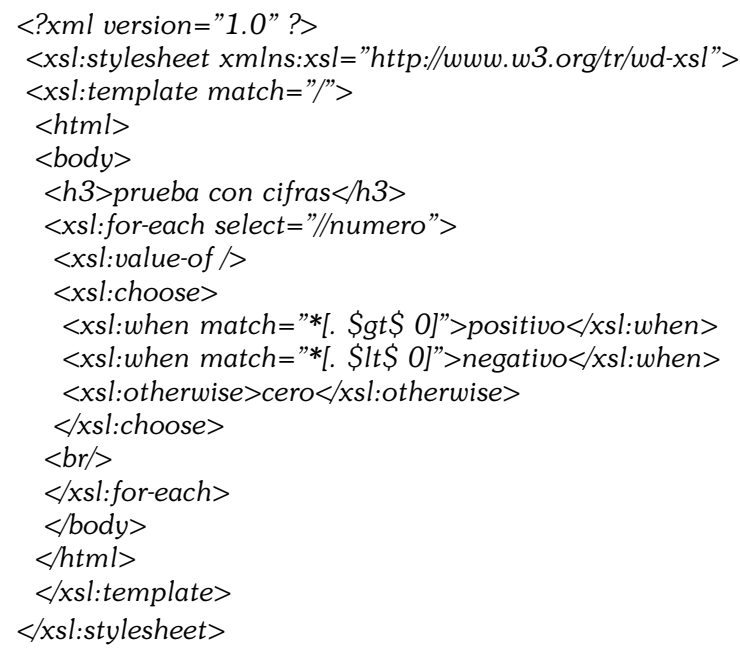

El template raíz crea la estructura básica de la página html que va a ser el resultado. Después se usa un loop $<x$ sl:for-each.. $>$ para iterar el programa sobre todos los elementos <numero $>$. El elemento $<x$ slt:valueof $>$ inserta en el resultado el valor actual del número que está siendo procesado, por lo que $\langle x$ sl:choose... $\rangle$ añade a ese valor el texto apropiado dependiendo de si es positivo, negativo o cero. El resultado de la hoja de estilo numeros.xsl actuando sobre el documento numeros.xml puede observarse en la figura 11 .

\section{«Xs/t proporciona un elemento especial que permite asociar scripting a los documentos $\mathrm{xml}$ definiéndolo en la hoja de estilo»}

La traducción del código $<x s l:$ when match $=$ "*[. $\$ g t \$ 0] ">$ positivo $<\mid x$ sl:when $>$ podría ser: "si cualquiera de los elementos seleccionados presenta un va- lor mayor que cero, entonces escribe el valor de ese elemento seguido de la cadena de caracteres positivo". Si asociamos cada parte de la expresión anterior con su correspondiente en el código resultaría algo así: "si cualquiera (por eso el '*') de los elementos seleccionados (<xsl:for-each select $=$ "//numero" $>$ ) presenta un valor (por eso el '.': el valor del elemento que se esté procesando en ese momento) mayor que cero ("* [. $\$ g t \$ 0] "(\$ g t \$=$ greater than $)(\$ l t \$=$ less than $))$, entonces escribe el valor de ese elemento (<xsl:value-of ।>) seguido de la cadena de caracteres positivo".

\section{«Es posible incluir scripts en las hojas de estilo xs/t, lo que resulta particularmente útil pues es actualmente la única técnica para incluirlos en docu- mentos xml puros»}

g.2. Añadir script a hojas de estilo xslt. Xslt proporciona distintos medios de manipular y presentar documentos xml en el navegador. Por lo tanto ¿se podría pensar que a partir de ahora es posible abandonar html y utilizar simplemente $\mathrm{xml}$ ? Una de los temas que se deben considerar es que en xml la etiqueta html $<$ script $>$ no significa nada. Para superar esta limitación, xslt proporciona un elemento especial que permite asociar scripting a los documentos xml definiéndolo en la hoja de estilo:

-Xsl:script

-Xsl:eval

1. Xsl:script. Este elemento define una sección script de la hoja de estilo que puede ejecutarse desde cualquier parte de la hoja de estilo simplemente invocándolo. <xsl:script...> acepta un atributo "langua$g e$ " que especifica el lenguaje de scripting que se va a usar:

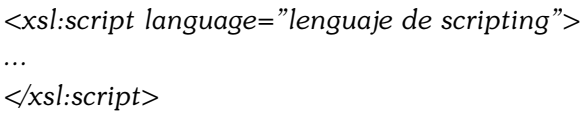

El siguiente ejemplo define una función que devuelve la hora y fecha actuales:

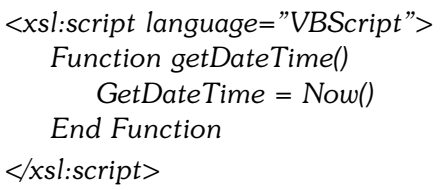

2. Xsl:eval. Se utiliza para evaluar una cadena de caracteres del script e insertar el resultado de la evaluación en el nuevo documento. También acepta un atributo "language":

$<x$ sl:eval language="lenguaje de scripting"> 
Si sus documentos son estratégicos para su compañía, también lo son para nosotros

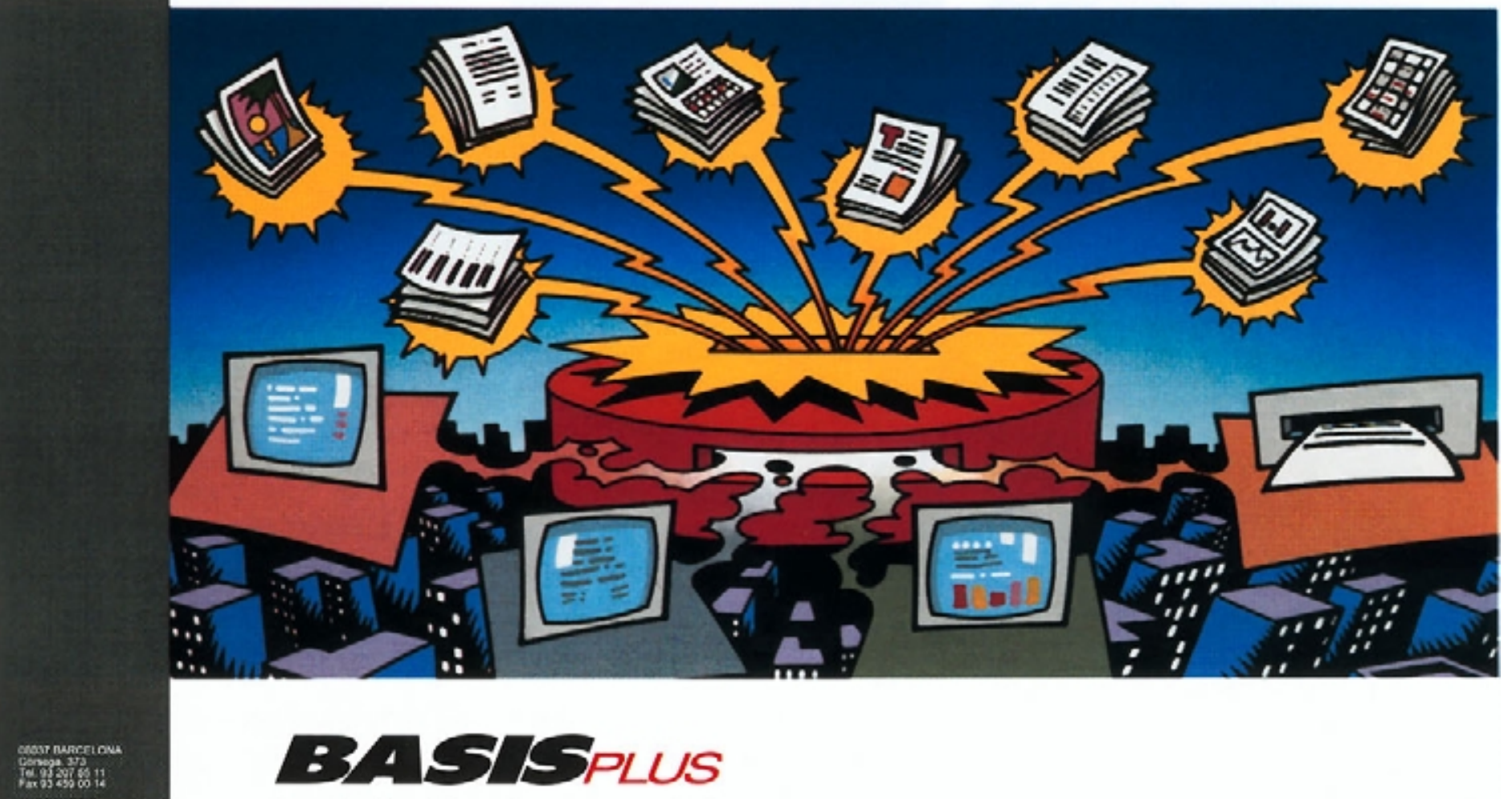

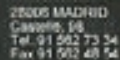

1500 Breso,

cि

jocos panplone

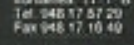

arovo var rncus

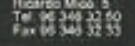

CEC

inim

है.

Marivir

tocm 0 in

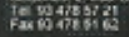

soc

conger

Centroshooktucus

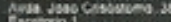

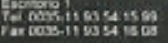

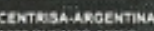

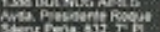

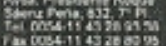

centrusex

vencon

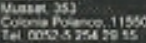

icosisestion

cenTRNen

Mations

250.0.

GINTRSA.COLOUNA

Grinfe DE 0009

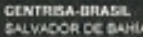

\section{La Solución Documental}

BASISplus es el único sistema de gestión de documentos que permite gestionar de un modo integrado toda la información que circula por su organización.

Con BASISplus su trabajo será más fácil, su tiempo más rentable y su información más segura y accesible.

\section{TECHLIBPLUS}

\section{La Gestión de Bibliotecas}

TECHLIBplus es una solución completa totalmente diseñada bajo entorno Web para gestionar los recursos de información y actualizar el trabajo diario de las bibliotecas.

Aplicaciones basadas en las tecnologias BASISplus y TECHLIBp/us:

Centros de Documentación

Sistemas de Gestión de Calidad

Gabinetes de Prensa

Difusión de Boletines Oficiales

Gestión de Expedientes

Normativa y Procedimientos

Producción Editorial

Gestion de Archivos

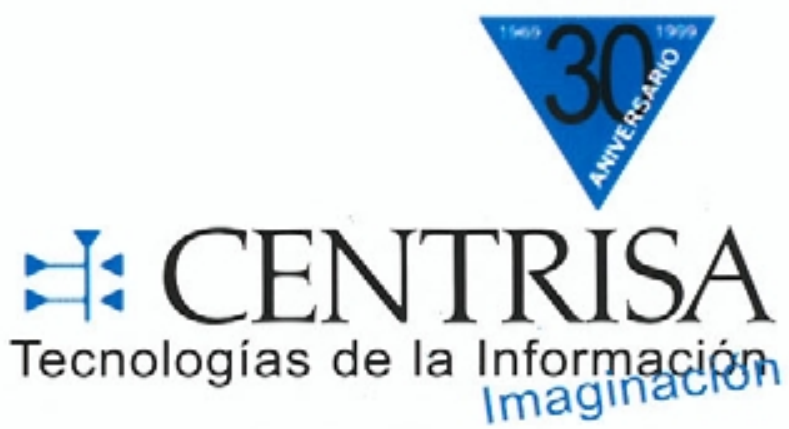

Más de 1 millón de usuarios,

2.500 instalaciones 2.500 instalacion 24 idiomas
en 75 paises y en 
$<\mid x s l: e v a l>$

Como ejemplo, se puede llamar a la función getDateTime() definida antes:

$<x$ sl:eval language $=$ "VBScript" $>$ getDateTime ()$<\mid x$ sl:eval $>$

El mismo código podría servir para llamar a la función now():

$<x$ sl:eval language="VBScript" $>$ Now ()$</$ xsl:eval $>$

\section{Conclusión}

En estas páginas se ha analizado una de las especificaciones del entorno xml con más proyección de futuro. Si se tuvieran que resumir las características de $x$ slt se podría hablar de su estructura jerárquica de forma y de su proximidad conceptual con la orientación a objetos.

\section{«Xs/t pretende situarse justo a medio camino, estructurando los objetos como xml, pero ges- tionando también su comporta- miento como en un lenguaje de programación»}

¿Cuál es la diferencia fundamental entre un lenguaje de programación orientado a objetos y un lenguaje de etiquetas como xml? La disparidad reside en el concepto de comportamiento de los objetos. Una parte importante de la orientación a objetos se basa en la especificación del comportamiento del objeto. Xml especifica sólo la estructura de los objetos (entiéndanse éstos el documento y sus elementos), excluyendo intencionadamente su comportamiento para dar a los desarrolladores la máxima flexibilidad a la hora de utilizar esos datos. Pues bien, xslt pretende situarse justo a medio camino, estructurando los objetos como xml, pero gestionando también su comportamiento como en un lenguaje de programación.

La flexibilidad, compatibilidad y vocación www de esta especificación son otras tres características muy importantes. Xslt es un tema muy amplio. Las posibilidades examinadas muy brevemente en este trabajo han sido simplemente: seleccionar, modificar, dar formato y presentar documentos xml transformándolos en código html. Hay que tener presente que las funcionalidades reales de aplicación de esta tecnología van mucho más allá. Por otra parte no se ha comentado nada sobre otros parsers (más sofisticados que IE5) como: Oracle xsl, Saxon, Xalan, xt, ixslt, Stylus o 4xslt simplemente para subrayar el hecho de que es una tecnología al alcance de todo aquel que pueda descargar en su sistema la última versión del navegador $I E$.
Las preguntas que quedan por hacerse son: ¿dónde sería más útil emplear esta tecnología?, ¿qué tipo de aplicaciones documentales podrían desarrollarse con ella? Las respuestas deberían darse en forma de proyectos concretos.

\section{Notas}

1. De ahí el nombre cascading style sheets, porque se aplican en cascada a un documento.

2. El "punto" es una unidad absoluta de dimensión usada en tipografía.

3. Definida por el autor del código.

4. Este ejemplo volverá a usarse en varias ocasiones a lo largo del trabajo.

5. Este bloque en realidad estará formado por el contenido de los descendientes del elemento <libro $>$, que no es más que una forma abstracta de agrupar ese contenido.

6. Aceptadas por la aplicación en la que el documento vaya a ser presentado. Generalmente un navegador.

7. También otros formatos.

8. Por el mismo razonamiento, el input del proceso xslt también podría ser una $D T D$, un esquema, etc., lo que nos da una idea aproximada del potencial de esta tecnología.

9. Generalmente elementos.

10. Xml permite a los desarrolladores crear sus propios lenguajes de etiquetas para sus propios proyectos. Estos lenguajes pueden compartirse con individuos que trabajen en el mismo tipo de proyecto a través de la web. Un ejemplo claro es xslt. El lenguaje de transformación xslt debe generar xml bien-formado que incluya etiquetas xslt. Por lo tanto se necesita un sistema para distinguir claramente cuándo esos elementos xml son en realidad "instrucciones" xslt y cuándo son simples elementos de salida (incluso si ambos elementos tienen el mismo nombre). Esa es la razón de ser de los namespaces, pues permiten que cada elemento pueda ser entendido en un entorno específico. Por ejemplo, los elementos xml que conciernen a las instrucciones de transformación xslt están en el namespace: http://www.w3.org/xsl/transform/1.0, mientras que los que sólo son elementos xml de salida están en: http://www.w3.org/xsl/format/1.0.

11. Because xslt style sheets are xml documents they should begin with the usual xml declaration.

12. El nodo raíz de book.xml era <listadelibros $></$ listadelibros $>$.

13. $<$ codigo/>, <categoria/ $>,<$ fecha_pub/> y <ventas $/>$.

14. <xslt:copy $>$ es particularmente útil cuando el documento fuente $X M$ contiene etiquetas html como $\langle b\rangle,\langle i\rangle$ o $\langle$ table $\rangle$. Usar el elemento $x$ slt:copy es como decir al procesador: "Trata estas etiquetas xml como si tuvieran su significado html habitual".

15. En xml el contenido de las secciones cdata o character data es tratado como texto plano. 